\title{
Hypofractionated Radiotherapy Based on Tomotherapy Application for Limited-Stage Small Cell Lung Cancer: Study Protocol for a Randomized, Controlled Trial
}

\section{Xiaofang Zhang}

Cancer Hospital of China Medical University: Liaoning Cancer Institute and Hospital

\section{Ziyi Xie}

Cancer Hospital of China Medical University: Liaoning Cancer Institute and Hospital

\section{Yingqiu Song}

Cancer Hospital of China Medical University: Liaoning Cancer Institute and Hospital

Chengsen Liu

Cancer Hospital of China Medical University: Liaoning Cancer Institute and Hospital

\section{Chenyu Wang}

Cancer Hospital of China Medical University: Liaoning Cancer Institute and Hospital

\section{Peng Zhao}

Cancer Hospital of China Medical University: Liaoning Cancer Institute and Hospital

\section{Bo Huang}

Cancer Hospital of China Medical University: Liaoning Cancer Institute and Hospital

\section{Lei He}

Cancer Hospital of China Medical University: Liaoning Cancer Institute and Hospital

Tianlu Wang ( $\nabla$ wangtianlu126@126.com )

Cancer Hospital of China Medical University https://orcid.org/0000-0002-9732-3441

\section{Study protocol}

Keywords: Tomotherapy, hypofractionated radiotherapy, limited-stage small cell lung cancer

Posted Date: June 30th, 2021

DOl: https://doi.org/10.21203/rs.3.rs-654247/v1

License: (c) (1) This work is licensed under a Creative Commons Attribution 4.0 International License. Read Full License 


\section{Abstract}

\section{Background}

Tomotherapy (TOMO), also known as spiral tomotherapy, offers better dose distribution, mild radiotherapy responses, shorter single-treatment times, and the benefits of timely adjustment of set errors. The overall treatment time of hypofractionated radiotherapy (HFRT) does not increase compared with that of conventional radiotherapy (Con-RT); thus, HFRT is considered the most promising and attractive alternative to standard segmentation. The effect of HFRT based on TOMO technology on limited-stage small cell lung cancer (LS-SCLC) patients requires further investigation.

\section{Methods}

The number of samples required was calculated using PASS software, and this randomized, controlled, prospective study will include 120 LS-SCLC patients randomized (1:1) to the experiment group (HFRT with TOMO) or the control group (Con-RT). The primary outcome is progression-free survival time after treatment with HFRT with TOMO. Secondary outcomes include acute and chronic adverse reactions, overall survival, tumor response (stable disease, progressive disease, complete response, and partial response), and quality of life. The follow-up duration will be 2 years. This is version 1.0 of protocol on April 1, 2021. The start of recruitment for this clinical trial is July 1,2020 , and the completion of recruitment is January 1, 2022.

\section{Discussion}

This new experimental study aims to explore the selection criteria of instruments during LS-SCLC radiotherapy and provide data theory support for the specified treatment specifications and guidelines. For problems that may arise in the research, we will solve them through statistical methods, such as differential analysis, and project funding support. The study is committed to providing better efficacy for patients with LS-SCLC.

\section{Trial registration}

This study is registered in the Chinese Clinical Trial Registry, with trial number ChiCTR1900027539 (date of registration: November 17, 2019). (URL:http://www.chictr.org.cn/edit.aspx?pid=45872\&htm=4)

\section{Background}

Small cell lung cancer (SCLC) accounts for approximately $15 \%$ of patients diagnosed with lung cancer, and it is an aggressive disease that has a short multiplication time, a high growth score, and is prone to widespread metastasis in the early stages [1-3]. Of those patients diagnosed with SCLC, $30 \%$ have limited-stage SCLC (LS-SCLC), which can be included in the area of tolerable radiation therapy. The standard treatment is etoposide (EP) combined with radiotherapy, and the National Comprehensive Cancer Network recommends LS-SCLC line 45-Gy/hypofractionated radiotherapy (HFRT) or 60-Gy 
conventional radiotherapy (Con-RT). Although the treatment is sensitive, the median survival remains 1624 months, which is far below clinical expectations [4]. Spiral tomotherapy (TOMO) is a novel method of intensity-modulated radiation therapy technology using a spiral $360^{\circ}$ radiation transmission system similar to that of spiral computed tomography (CT) scanning. The effects of spiral TOMO are often utilized for the treatment of a variety of diseases $[5,6]$. Furthermore, it has better dose distribution, mild radiation therapy response, shorter single-treatment time, and the advantages of timely adjustment of set error, resulting in better dose adaptability to the target and reduction of the impact on breathing and heartbeat and of the other effects caused by the error [7]. By quickly opening and closing the leaves in a collimator that rotates around the patient, TOMO can carve radiation doses into complex-shaped tumor areas while avoiding radiation to normal organ [8]. In addition, HFRT may be the best example of the 2-Gy routine classical grading changes using a daily single component, including two or more smaller doses to increase the dose per day in order to reduce tumor cell reaggregation between two consecutive small doses, thereby increasing the total dose of administration but not the risk of long-term toxicity [9]. The most common treatment option is to perform conventional radiation therapy (Con-RT) in batches at 2.0 Gy/day for 5 days a week, with a duration of 6-7 weeks [10]. Compared with Con-RT, the overall treatment time of HFRT does not increase and is considered the most promising and attractive alternative to standard segmentation. The basic principle of HFRT is to reduce late toxicity by using a smaller fraction size and to allow sufficient time for adequate repair of sub-fatal injuries between fractions. This strategy allows for the delivery of a higher total radiation dose to enhance tumor control without correspondingly increasing late-stage toxicity [11]. Therefore, it is necessary to investigate if the use of HFRT with TOMO may increase the percentage of local area control of LS-SCLC without increasing acute or chronic toxicity and thus lead to improved parameter indicators such as progression-free survival (PFS) time extension.

\section{Methods And Design}

\section{Design}

The novel application of HFRT using TOMO technology will be performed in a randomized, controlled, prospective, and safety study using the American ADAC Pinnacle treatment planning system (ADAC Laboratories, Milpitas, CA, USA) in 120 enrolled LS-SCLC patients. The number of samples was calculated using IBM SPSS Statistics 19 software. The LS-SCLC patients will be randomized (1:1) to the experiment group (HFRT with TOMO) or the control group (Con-RT) using a random number table. This study does not use the blinded method because HFRT with TOMO and Con-RT have a large difference in the implementation process, which makes blinding difficult. However, the deviation caused by not using the blinded method has no significant effect on the patients' outcome assessment. In this therapeutic study, LS-SCLC is defined as a lesion confined to one side of the thoracic cavity, mediastinum, anterior oblique muscle, or supraclavicular lymph nodes, but has no obvious superior vena cava compression, vocal cord paralysis, or pleural effusion. Patients who meet the inclusion criteria will be grouped by randomized parallel control into the HFRT with TOMO and Con-RT groups according to 
randomly assigned results. The study will be carried out at the Liaoning Cancer Hospital Medical Center, a tertiary medical center in Liaoning, China.

\section{Objectives}

This study aims to assess the efficacy and safety of HFRT with TOMO for the treatment of LS-SCLC. We will record the weekly physical examinations, hematological examinations, and liver and kidney function test. The main purpose of this effectiveness study is to determine whether a follow-up trial to evaluate efficacy and safety in a larger cohort would be feasible.

\section{Primary Study Outcome}

The primary outcome is PFS time after radiotherapy, which refers to the number of months between the initiation of radiotherapy and the first evidence of progressive disease as defined by the Response Evaluation Criteria In Solid Tumors criteria.

\section{Secondary Study Outcomes}

\section{Acute and chronic adverse reactions}

The secondary study outcomes are acute and chronic adverse reactions, which will be evaluated according to the Radiation Therapy Oncology Group/European Organisation for Research and Treatment of Cancer standard, which define chronic adverse reactions as reactions that occur after radiotherapy or that last for $>90$ days).

\section{Overall survival}

According to the Tumor Lymph Node Metastasis standard established by the American Joint Committee on Cancer and the International Cancer Coalition Cancer Control, overall survival (OS) is the number of months between the initiation of radiotherapy and the last follow-up or death.

\section{Tumor response}

The tumor responses are progressive disease (PD), stable disease (SD), complete response (CR), and partial response (PR). PD refers to $\geq 20 \%$ increase in the sum of the maximum diameter of target lesions or the appearance of new lesions. SD refers to the sum of the maximum diameter of the target lesions that did not reach PR but increased to reach PD. CR is the complete disappearance of the tumor after radiotherapy. $\mathrm{PR}$ is the product of the reduction of the maximum diameter and maximum vertical diameter of the tumor by $50 \%$ after radiotherapy, and no increase in other lesions, which lasts more than 1 month.

\section{Quality of life}


The assessment of quality of life (QoL) includes 60 questionnaires on the QoL of LS-SCLC patients at baseline, 4 weeks, 3 months, 6 months, 12 months, and 24 months after HFRT with TOMO and after ConRT.

\section{Hypotheses}

Our primary hypothesis is that the use of HFRT with TOMO will increase clinical PFS time for approximately 3-6 months because of better tumor control [12-14]. The PFS and OS rates of patients in this study will be compared with those who are eligible to participate in the study but are receiving standard radiation therapy. If necessary, PFS data can also be compared with data of the historical cohort of patients receiving treatment in our center. Our secondary hypothesis is that the OS rate will increase by approximately $5 \%$ because of better local tumor control [15]. In addition, we hypothesize that relevant acute and chronic adverse reactions will decrease and that QoL will improve.

\section{Population}

The study population included 120 patients diagnosed with LS-SCLC. The inclusion and exclusion criteria are listed in Table 1.

Table 1 Inclusion and exclusion criteria

\section{Inclusion criteria}

- Age $\geq 18$ years

- Stage I-III small cell lung cancer

- No serious cardiopulmonary dysfunction

- Blood routine test result and liver, kidney and heart function are basically normal

\section{Exclusion criteria}

- Obvious mental disorders or intolerance to radiotherapy treatment

- Have difficulty following doctor's orders in completing relevant examinations and treatments

- Karnofsky Performance Score $\leq 70$ points

- Severe ventilation dysfunction of lung function

- Bone marrow suppression above grade

- Moderate to severe cardiac insufficiency

The eligibility of patients who are candidates for HFRT with TOMO or Con-RT group will be discussed upfront by the expert group. The expert panel includes three chief physicians, four attending physicians, and two interventional radiologists.

\section{Study Outline}

TOMO is a new type of image-guided intensity-modulated radiation therapy system that uses CT scanning and fan-shaped spiral irradiation. TOMO, which uses imaging-guided radiotherapy and adaptive radiotherapy as its main function, has been used in radiotherapy of chest tumors for more than 10 years and has obtained good clinical efficacy and safety. 


\section{Required equipment and technical support}

The Radiotherapy Department of Liaoning Provincial Cancer Hospital has started TOMO treatment since November 2015 and has established a database. Imaging-guided radiotherapy is performed with the ADAC Pinnacle treatment planning system, radiotherapy special positioning body frame, polymer lowtemperature hydrolyzed plastic neck and shoulder membrane, and laser light positioning (for special external positioning system). This project is guided by senior physicians who have been engaged in this work for many years, and excellent physicists provide technical support. The TOMO treatment equipment and radiotherapy planning system produced in the United States are already available. Our department has successfully completed precise radiotherapy based on TOMO technology in many cases.

\section{CT positioning scanning method}

The patient lies on a special body frame for radiotherapy, and the polymer low-temperature hydrolyzed plastic body film is used to fix the position. The laser is positioned on the midline of the chest and on both sides (for a special external positioning system), and the corresponding reference points are marked on the body film and body surface. Lead pellets are placed for marking. With the patient calmly breathing, spiral CT scans the chest continuously with a pitch of $3 \mathrm{~mm}$ and transmits the data to the ADAC Pinnacle ${ }^{3}$ TPS workstation via the network. Each patient routinely undergoes large-aperture CT positioning, with the target area delineated according to enhanced CT images.

\section{Formulation and implementation of TOMO radiotherapy plans}

A radiotherapy department chief physician and an experienced image diagnosis physician outline the radiotherapy target area on the CT image. The gross tumor volume in the tumor area, set as $0.8 \mathrm{~cm}$, is designated as the clinical target area, and the clinical tumor volume is set as $0.5 \mathrm{~cm}$; the tumor motion range is used as the planned target area planning tumor volume. A medical linear accelerator CT scan is used to correct the positioning error from a three-dimensional perspective to ensure the accuracy of the treatment and to ensure that it can be safely implemented during conventional or large-scale radiotherapy. The TOMO radiotherapy plan can be formulated on the Pinnacle ${ }^{3}$ treatment planning system. A VARIAN high-energy electron beam accelerator 6-MV X ray (Varian Medical Systems, Palo-Alto, CA, USA) is used for irradiating the tomographic intensity-modulated irradiation field.

\section{Treatment plan}

Patients who meet the inclusion criteria will be given radiotherapy. In the HFRT with TOMO group, the prescribed dose of the planning tumor volume is $1.5 \mathrm{~Gy}$, twice a day, and the interval between each radiotherapy is at least 12 hours. A total of 30 radiotherapy treatments are performed. In the Con-RT group, the prescribed dose of the planning tumor volume is $2 \mathrm{~Gy}$, once a day. A total of 30 radiotherapy treatments are performed. When the radiotherapy reaches 30 or $40 \mathrm{~Gy} / 20$ fractions or times, the largeaperture $\mathrm{CT}$ is reset and the two examination images are fused. Based on the review of the CT images, the target area is redrawn to formulate a treatment plan based on the second examination 
images. Precise radiotherapy is continued until the prescription dose accumulates to $45 \mathrm{~Gy} / 30$ times. Concurrent EP chemotherapy during radiotherapy (EP $50 \mathrm{mg} / \mathrm{m}^{2}$, days $1-5$, cisplatin $25 \mathrm{mg} / \mathrm{m}^{2}$, days $1-3$ ) is performed for 1 cycle, with an EP program for 21 days as a course; simultaneous chemotherapy is performed for 1 cycle and adjuvant five cycles, for a total of 6 cycles of chemotherapy. CT is reviewed once every 2 cycles of chemotherapy and merged with the previous examination to outline the residual tumor area [16-18]. After the end of chemotherapy, the CT should be rechecked every 2 months and merged with the previous examination. The residual tumor area should be delineated until the target lesion has completely subsided. Follow-up is performed until the tumor recurs locally.

\section{Post-radiotherapy follow-up}

The first follow-up and comprehensive assessment of the effectiveness of treatment will be performed in the fourth week after the completion of HFRT with TOMO and Con-RT. A review will be conducted every 3 months for the next 2 years, including physical examination, enhanced CT scan, blood routine test, liver and kidney functions, and QoL; if necessary, brain-enhanced magnetic resonance imaging and bone imaging examination will be performed to assess the effectiveness of different radiotherapy options.

\section{Data analysis}

After the last patient completes follow-up, the trial committee will assess the occurrence of the primary and secondary end points. The primary end point will be expressed as PFS time (months) after treatment. The OS will also be expressed as months. For statistical assumptions, the alpha value will be set to $P<0.05$. The data will be analyzed by the Social Science Statistical Software Package (IBM Corp., Armonk, NY, USA). Appropriate parametric and non-parametric statistical tests will be conducted to analyze the statistical data of different patient conditions and draw meaningful conclusions. If necessary, multiple comparisons and corrections will be made. Tumor response data (PD, SD, CR, and PR) will be expressed as a percentage.

\section{Discussion}

The study will assess the efficacy and safety of HFRT with TOMO for the treatment of LS-SCLC. To the best of our knowledge, no one has ever implemented a similar project. Its value lies in exploration of the selection criteria of instruments during LS-SCLC radiotherapy and collection and presentation of data theory support for the specified treatment specifications and guidelines. This study can fill the gap in the comparison of the efficacy of the experiment group (HFRT with TOMO) and the control group (Con-RT) in LS-SCLC. By breaking through traditional treatment methods and formulating new clinical treatment plans based on actual clinical problems, we expect to produce high-quality research results, which will ultimately benefit LS-SCLC patients. During the implementation of the study, we may encounter problems, including individual patient differences, and dropouts from the treatment course due to price, and other reasons. We will attempt to solve such problems through statistical methods, such as differential 
analysis, and project funding support. We intend to collect enough statistical sample numbers to ensure the smooth progress of the experiment. In the course of study development, opportunities and challenges coexist, and the details will be constantly reflected on and improved in actual implementation. The novelty of the design of this study is that it proposes a new treatment standard based on the new technology of TOMO, which provides a new method for the treatment of patients with LS-SCLC as wells as those with similar diseases.

\section{Trial status}

The recruitment of participants started on $1 \mathrm{Jul} 2020$ and the trial is expected to continue until Jan 2023.

On submission for publication, version 1.0 of the protocol was being used. Nov 2020

\section{Abbreviations}

Con-RT, conventional radiotherapy

$\mathrm{CR}$, complete response

$\mathrm{CT}$, computed tomography

EP, etoposide

HFRT, hypofractionated radiotherapy

LS-SCLC, limited-stage small cell lung cancer

OS, overall survival

$P D$, progressive disease

PFS, progression-free survival time

$\mathrm{PR}$, partial response

QoL, quality of life

$\mathrm{SD}$, stable disease

TOMO, tomotherapy

\section{Declarations}

\section{Ethics approval and consent to participate}


The ethics approval received by Medical Ethics Committee of Liaoning Cancer Hospital and the number is 2020X0101. Prior to screening, all participants should sign an informed consent form.

\section{Consent for publication}

Not applicable.

\section{Availability of data and materials}

The data of this study is available from the corresponding author upon reasonable request.

\section{Competing interests}

The authors declare that they have no competing interests.

\section{Funding}

Funding for a three-year doctorate scholarship period (2020-2023) was received from the National Cancer Center Special Oncology Research (NCC2017A08) and the Shenyang Major Scientific Research Projects (No.191124090) in order to conduct and analyze data from this ongoing study.

\section{Authors' contributions}

All authors participated in the development and the design of the study. Ziyi Xie and Chengsen Liu applied for ethical consent. Xiaofang Zhang wrote the first version of the manuscript. Yingqiu Song and Chenyu Wang is responsible for the data management and statistical considerations of this project. Peng Zhao work as a therapist in this clinical experiment. Lei He strictly controlled the quality of the patient's radiation treatment process. Bo Huang provides pathological reports to patients in clinical trials. Tianlu Wang critically revised the manuscript. All authors read and approved the final version of the manuscript.

\section{Acknowledgements}

We are very grateful to the participants who participated in the study because they spend precious time participating in the study.

\section{Authors' information}

${ }^{1}$ Department of Radiotherapy, Cancer Hospital of China Medical University, Shenyang, China; ${ }^{2}$ China Medical University, Shenyang, China; ${ }^{3}$ Department of Information Management, Cancer Hospital of China Medical University, Shenyang, China; ${ }^{4}$ Department of Pathology, Cancer Hospital of China Medical University, Shenyang, China; ${ }^{5}$ Physical Laboratory in Charge, Department of Radiotherapy Department, Cancer Hospital of China Medical University, Shenyang, China.

\section{References}


1. Miller K, Goding Sauer A, Ortiz A, Fedewa S, Pinheiro P, Tortolero-Luna G, et al. Cancer Statistics for Hispanics/Latinos 2018 CA: a cancer journal for clinicians. 2018;68(6):425-45.

2. Amini A, Byers L, Welsh J, Komaki R. Progress in the management of limited-stage small cell lung cancer. Cancer. 2014;120(6):790-8.

3. van Meerbeeck J, Fennell D, De Ruysscher D. Small-cell lung cancer. Lancet. 2011;378(9804):174155.

4. Turrisi A, Kim K, Blum R, Sause W, Livingston R, Komaki R, et al. Twice-daily compared with oncedaily thoracic radiotherapy in limited small-cell lung cancer treated concurrently with cisplatin and etoposide. N Engl J Med. 1999;340(4):265-71.

5. Sterzing F, Schubert K, Sroka-Perez G, Kalz J, Debus J, Herfarth K. Helical tomotherapy. Experiences of the first 150 patients in Heidelberg. Strahlentherapie und Onkologie: Organ der Deutschen Rontgengesellschaft [et al]. 2008;184(1):8-14.

6. Uhl M, Sterzing F, Habl G, Schubert K, Holger H, Debus J, et al. Breast cancer and funnel chest. Comparing helical tomotherapy and three-dimensional conformal radiotherapy with regard to the shape of pectus excavatum. Strahlentherapie Onkologie: Organ der Deutschen Rontgengesellschaft [et al]. 2012;188(2):127-35.

7. Li J, Zhao Z, Wu X, Yao J, Ma L, Ye R, et al. Bevacizumab plus cisplatin and helical tomotherapy in treatment of locally advanced nasopharyngeal carcinoma. OncoTargets therapy. 2015;8:1315-9.

8. Balog J, Mackie T, Pearson D, Hui S, Paliwal B, Jeraj R. Benchmarking beam alignment for a clinical helical tomotherapy device. Medical physics. 2003;30(6):1118-27.

9. Beck-Bornholdt H, Dubben H, Liertz-Petersen C, Willers H. Hyperfractionation: where do we stand? Radiotherapy oncology: journal of the European Society for Therapeutic Radiology Oncology. 1997;43(1):1-21.

10. Pignon J, Bourhis J, Domenge C, Designé L. Chemotherapy added to locoregional treatment for head and neck squamous-cell carcinoma: three meta-analyses of updated individual data. MACH-NC Collaborative Group. Meta-Analysis of Chemotherapy on Head and Neck Cancer. Lancet. 2000;355(9208):949-55.

11. Stuschke $M$, Thames $H$. Fractionation sensitivities and dose-control relations of head and neck carcinomas: analysis of the randomized hyperfractionation trials. Radiotherapy oncology: journal of the European Society for Therapeutic Radiology Oncology. 1999;51(2):113-21.

12. Zwicker F, Swartman B, Roeder F, Sterzing F, Hauswald H, Thieke $C$, et al. In vivo measurement of dose distribution in patients' lymphocytes: helical tomotherapy versus step-and-shoot IMRT in prostate cancer. J Radiat Res. 2015;56(2):239-47.

13. Balana C, Vaz M, Sepúlveda J, Mesia C, Del Barco S, Pineda E, et al. A phase II randomized, multicenter, open-label trial of continuing adjuvant temozolomide beyond six cycles in patients with glioblastoma (GEINO 14 - 01). Neuro-oncology. 2020.

14. Jablonska P, Diez-Valle R, Gállego Pérez-Larraya J, Moreno-Jiménez M, Idoate M, Arbea L, et al. Correction: Hypofractionated radiation therapy and temozolomide in patients with glioblastoma and 
poor prognostic factors. A prospective, single-institution experience. PloS one. 2019;14(7):e0219614.

15. Malmström A, Grønberg B, Marosi C, Stupp R, Frappaz D, Schultz H, et al. Temozolomide versus standard 6-week radiotherapy versus hypofractionated radiotherapy in patients older than 60 years with glioblastoma: the Nordic randomised, phase 3 trial. The Lancet Oncology. 2012;13(9):916-26.

16. Navarria P, Pessina F, Franzese C, Tomatis S, Perrino M, Cozzi L, et al. Hypofractionated radiation therapy (HFRT) versus conventional fractionated radiation therapy (CRT) for newly diagnosed glioblastoma patients. A propensity score matched analysis. Radiotherapy oncology: journal of the European Society for Therapeutic Radiology Oncology. 2018;127(1):108-13.

17. locolano M, Wild A, Hannum M, Zhang Z, Simone C, Gelblum D, et al. Hypofractionated vs. conventional radiation therapy for stage III non-small cell lung cancer treated without chemotherapy. Acta oncologica (Stockholm Sweden). 2020;59(2):164-70.

18. Ganju R, TenNapel M, Chen A, Mitchell M. Impact of Peer Review on Use of Hypofractionated Regimens for Early-Stage Breast Cancer for Patients at a Tertiary Care Academic Medical Center and Its Community-Based Affiliates. Journal of oncology practice. 2019;15(2):e153-e61.

\section{Figures}




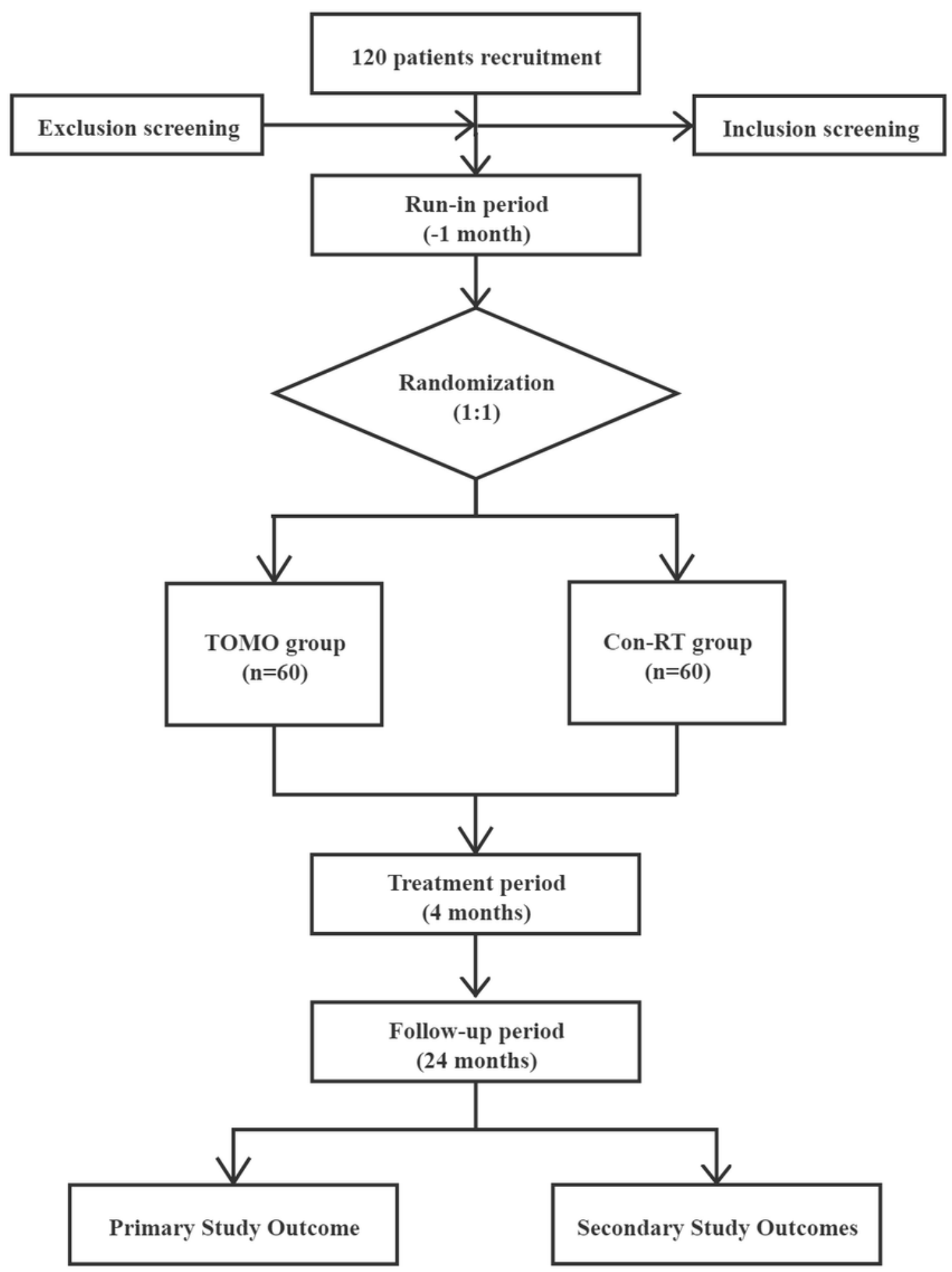

\section{Figure 1}

Flowchart of the study procedure: 120 eligible subjects will be randomly allocated into the TOMO group and Con-RT group in a 1:1 ratio; TOMO, tomotherapy; Con-RT, conventional radiotherapy. 


\begin{tabular}{|c|c|c|c|c|c|c|c|}
\hline \multirow[b]{3}{*}{ Time point } & \multicolumn{7}{|c|}{ Study Period } \\
\hline & \multicolumn{2}{|c|}{ Run-in } & \multicolumn{4}{|c|}{ Treatment } & \multirow{2}{*}{$\begin{array}{c}\text { Follow-up } \\
28 M\end{array}$} \\
\hline & $-1 M$ & $\mathbf{0 M}$ & $1 M$ & $2 M$ & $3 M$ & $4 M$ & \\
\hline Visit & Visit1 & Visit2 & Visit3 & Visit4 & Visit5 & Visit6 & Visit7 \\
\hline \multicolumn{8}{|l|}{ Enrollment } \\
\hline Eligibility screen & $\sqrt{ }$ & $\sqrt{ }$ & & & & & \\
\hline Informed consent & $\sqrt{ }$ & & & & & & \\
\hline Demographics & $\sqrt{ }$ & & & & & & \\
\hline Randomization & & $\sqrt{ }$ & & & & & \\
\hline \multicolumn{8}{|l|}{ Intervention } \\
\hline \multicolumn{8}{|l|}{ TOMO group } \\
\hline \multicolumn{8}{|l|}{ Con-RT group } \\
\hline \multicolumn{8}{|l|}{ Assessments } \\
\hline Study diary & & $\sqrt{ }$ & $\sqrt{ }$ & $\sqrt{ }$ & $\sqrt{ }$ & $\sqrt{ }$ & \\
\hline PFS & & & & & & $v$ & $v$ \\
\hline $\begin{array}{l}\text { Acute and chronic } \\
\text { adverse reactions }\end{array}$ & & $\sqrt{ }$ & $\sqrt{ }$ & $\sqrt{ }$ & $\sqrt{ }$ & $\sqrt{ }$ & $\sqrt{ }$ \\
\hline Overall survival & & & & & & $v$ & $\checkmark$ \\
\hline Tumor response & & & $\sqrt{ }$ & $\sqrt{ }$ & $\sqrt{ }$ & $\sqrt{ }$ & $\sqrt{ }$ \\
\hline Quality of life & & $\sqrt{ }$ & $\sqrt{ }$ & $\sqrt{ }$ & $\sqrt{ }$ & $\sqrt{ }$ & $\sqrt{ }$ \\
\hline $\begin{array}{c}\text { Whole genome } \\
\text { analysis }\end{array}$ & & $\sqrt{ }$ & & & & $\checkmark$ & \\
\hline
\end{tabular}

\section{Figure 2}

Recommended content for the schedule of enrolment, interventions, and assessments.

\section{Supplementary Files}

This is a list of supplementary files associated with this preprint. Click to download.

- renamed7e1bc.doc 